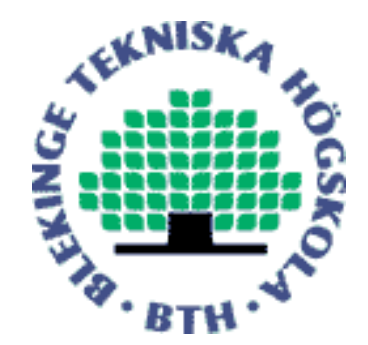

Copyright @ 2012 IEEE.

Citation for the published paper:

Title:

In press: Unified Analysis of Transmit Antenna Selection in MIMO Multi-Relay Networks

Author:

Phee Lep Yeoh, Maged Elkashlan, Nan Yang, Daniel B. da Costa, Quang Trung Duong

Journal:

IEEE Trans on Vehicular Technology

Year:

2012

Vol:

Issue:

Pagination:

URL/DOI to the paper:

Unified Analysis of Transmit Antenna Selection in MIMO Multi-Relay Ne

This material is posted here with permission of the IEEE. Such permission of the IEEE does not in any way imply IEEE endorsement of any of BTH's products or services Internal or personal use of this material is permitted. However, permission to reprint/republish this material for advertising or promotional purposes or for creating new collective works for resale or redistribution must be obtained from the IEEE by sending a blank email message to pubs-permissions@ieee.org.

By choosing to view this document, you agree to all provisions of the copyright laws protecting it. 


\title{
Unified Analysis of Transmit Antenna Selection in MIMO Multi-Relay Networks
}

\author{
Phee Lep Yeoh, Member, IEEE, Maged Elkashlan, Member, IEEE, Nan Yang, Member, IEEE, \\ Daniel B. da Costa, Member, IEEE, and Trung Q. Duong, Member, IEEE.
}

\begin{abstract}
We present a unified asymptotic framework for transmit antenna selection in multiple-input multiple-output (MIMO) multi-relay networks with Rician, Nakagami- $m$, Weibull, and Generalized- $\mathcal{K}$ fading channels. We apply this framework to derive new closed-form expressions for the outage probability and symbol error rate (SER) of amplify-andforward relaying in MIMO multi-relay networks with two distinct protocols: 1) transmit antenna selection with receiver maximalratio combining (TAS/MRC), and 2) transmit antenna selection with receiver selection combining (TAS/SC). Based on these expressions, the diversity order and the array gain with $M$-ary phase shift keying and $M$-ary quadrature amplitude modulation are derived. We corroborate that the diversity order only depends on the fading distribution and the number of diversity branches, whereas the array gain depends on the fading distribution, the modulation format, the number of diversity branches, and the average per-hop signal-to-noise ratios (SNRs). We highlight that the diversity order of TAS/MRC is the same as TAS/SC, regardless of the underlying fading distribution. As such, we explicitly characterize the SNR gap between TAS/MRC and TAS/SC as the ratio of their respective array gains. An interesting observation is reached that for equal per-hop SNRs, the SNR gap between the two protocols is independent of the number of relays.
\end{abstract}

Index Terms-MIMO, relays, TAS/MRC, TAS/SC

\section{INTRODUCTION}

W IRELESS relaying is currently under consideration in 3GPP LTE and IEEE $802.16 \mathrm{~m}$ as a cost-effective application to support high data rates at the cell-edge [1]. Indeed, the deployment of energy efficient relays is acknowledged as a key strategy for coverage extension and capacity enhancement in future heterogenous wireless networks [2]. In pursuit of peak data rates between $100 \mathrm{Mbps}$ and $1 \mathrm{Gbps}$,

Copyright (c) 2012 IEEE. Personal use of this material is permitted. However, permission to use this material for any other purposes must be obtained from the IEEE by sending a request to pubs-permissions @ieee.org.

Manuscript received May 8, 2012; revised September 05, 2012; accepted October 29, 2012. The review of this paper was coordinated by Prof. Yahong Zheng.

P. L. Yeoh is with the Department of Electrical and Electronic Engineering, University of Melbourne, VIC 3010, Australia (e-mail: phee.yeoh@unimelb.edu.au).

M. Elkashlan is with the School of Electronic Engineering and Computer Science, Queen Mary, University of London, London E1 4NS, UK (email: maged.elkashlan@eecs.qmul.ac.uk).

N. Yang is with the School of Electrical Engineering and Telecommunications, The University of New South Wales, Sydney, NSW 2052, Australia (email: nan.yang@unsw.edu.au).

D. B. da Costa is with Federal University of Ceará (UFC), Sobral, CE, Brazil (email: danielbcosta@ieee.org).

T. Q. Duong is with Blekinge Institute of Technology, Karlskrona, Sweden (email: quang.trung.duong@bth.se).

Digital Object Identifier xx.xxxx/TVT.2012.xxxx recent standardization efforts have considered multiple-input multiple-output (MIMO) processing in relay networks with up to 4 transmit/receive antennas at the base station, relay node, and user equipment [3].

MIMO relaying has attracted widespread interest in the recent literature. One approach to MIMO relaying is transmit beamforming which activates all the antennas at the source and the relays [4, 5]. In [4], the MIMO relay broadcast channel is analyzed and a low complexity beamforming optimization algorithm is proposed. Practical beamforming protocols are developed in [5] that are robust to limited and imperfect channel state information. An economical alternative to beamforming is antenna selection which activates a single transmit and/or receive antenna. Antenna selection offers reduced feedback overhead, low implementation complexity, and low signal processing cost while preserving the diversity benefits of MIMO.

In this letter, we present a unified analytical framework for MIMO multi-relay networks with two distinct antenna selection protocols, namely: 1) transmit antenna selection with receive maximal-ratio combining (TAS/MRC), and 2) transmit antenna selection with receiver selection combining (TAS/SC). TAS/MRC and TAS/SC relaying have been previously considered in single-relay networks [6-9]. In TAS/MRC relaying, a single transmit antenna at the source and the relay offering the highest received signal-to-noise ratio (SNR) are selected, and all the receive antennas at the relay and destination are combined with MRC [6,7]. In TAS/SC relaying, a single antenna pair that achieves the highest received SNR is selected in the source-to-relay link and the relay-to-destination link [8, 9]. The asymptotic analysis of single antenna multi-relay networks over Rician and Nakagami- $m$ fading channels was previously presented in $[10,11]$. Recently, the performance of relaying in other fading distributions such as Rician, Weibull and Generalized $\mathcal{K}$ K fading has also come into consideration [12]. Indeed, these distributions have been proven to offer a close fit to experimental measurements in indoor and outdoor fading environments $[13,14]$. In this paper, we consider TAS/MRC and TAS/SC relaying in a wide range of important fading channels including Rician, Nakagami- $m$, Weibull, and Generalized- $\mathcal{K}$.

Our aim is to address the following fundamental questions: 1) How much improvement does TAS/MRC offer relative to TAS/SC in MIMO multi-relay networks? and 2) What are the explicit network parameters that determine this improvement with higher order modulations and generalized fading environments? Our response is in the form of new easy-to- 
calculate asymptotic expressions for the outage probability and SER of MIMO multi-relay networks with $M$-ary phase shift keying ( $M$-PSK) and $M$-ary quadrature amplitude modulation ( $M$-QAM). Our results are valid for arbitrary $L$ amplifyand-forward (AF) relays and $N_{\mathrm{S}}, N_{\mathrm{R}}$, and $N_{\mathrm{D}}$ antennas at the source, relays, and destination, respectively. Using these expressions, we explicitly characterize the diversity orders TAS/MRC and TAS/SC for Rician, Nakagami- $m$, Weibull, and Generalized- $\mathcal{K}$ fading channels. We confirm that the two protocols exhibit the same diversity order for each of the aforementioned fading channels. Due to this, one can conclude that the SNR gap between TAS/MRC and TAS/SC relaying is strictly characterized by their array gains. We highlight the interesting observation that the SNR gap is independent of $L$ for equal per-hop SNRs. We concisely express this SNR gap in terms of the fading parameters and the number of antennas $N_{\mathrm{S}}, N_{\mathrm{R}}$ and $N_{\mathrm{D}}$.

\section{PRotocol Description}

In this section, we detail two transmit antenna selection protocols for use in MIMO multi-relay networks where the source transmits to the destination with the aid of $L$ parallel AF relays. When considering multiple relays, we adopt opportunistic relaying [11] such that the relay with the highest end-to-end SNR is selected to transmit. We note that the selected source antenna may be different for each relay. In the following, $\mathcal{E}_{\mathrm{S}}$ is the source transmit power, $\mathcal{E}_{\mathrm{R}}$ is the relay transmit power, $\mathcal{N}_{0}$ is the variance of the additive white Gaussian noise (AWGN), $\|\cdot\|$ is the Euclidean norm, $|\cdot|$ is the absolute value, $(\cdot)^{\dagger}$ is the conjugate transpose, $\mathbf{I}_{N}$ is the $N \times N$ identity matrix, and $\mathbf{E}[\cdot]$ denotes the expectation.

\section{A. TAS/MRC Relaying}

In the source-to-relay link, a single antenna from $N_{\mathrm{S}}$ antennas at the source is selected to maximize the received SNR at the relay. In doing so, all the $N_{\mathrm{R}}$ antennas at the relay are combined with MRC. We denote $\mathbf{h}_{i}^{j}$ as the channel vector from the $j$-th transmit antenna at the source to all the receive antennas at the relay. As such, the received signal vector at the $i$-th relay is $\mathbf{y}_{\mathrm{SR}_{\mathrm{i}}}=\sqrt{\mathcal{E}_{\mathrm{S}}} \mathbf{h}_{i}^{\text {max }} x+\mathbf{n}_{\mathrm{R}}$, where $x$ is the transmit symbol, $\mathbf{n}_{\mathrm{R}}$ is the $N_{\mathrm{R}} \times 1$ AWGN vector satisfying $\mathbf{E}\left[\mathbf{n}_{\mathrm{R}} \mathbf{n}_{\mathrm{R}}^{\dagger}\right]=\mathbf{I}_{N_{\mathrm{R}}} \mathcal{N}_{0}$, and $\left\|\mathbf{h}_{i}^{\max }\right\|=\max _{1 \leq j \leq N_{\mathrm{S}}}\left\{\left\|\mathbf{h}_{i}^{j}\right\|\right\}$ denotes the largest channel vector between the source and the $i$-th relay.

In the relay-to-destination link, a single antenna from $N_{\mathrm{R}}$ antennas at the $i$-th relay is selected to maximize the received SNR at the destination. In doing so, all the $N_{\mathrm{D}}$ antennas at the destination are MRC combined. Prior to forwarding, a variable gain of $\alpha=1 / \sqrt{\mathcal{E}_{\mathrm{S}}\left\|\mathbf{h}_{i}^{\max }\right\|^{4}+\mathcal{N}_{0}\left\|\mathbf{h}_{i}^{\max }\right\|^{2}}$ is applied to counteract the fading in the source-to-relay link. We denote $\mathbf{g}_{i}^{j}$ as the channel vector from the $j$-th transmit antenna at the relay to all the receive antennas at the destination. As such, the received signal vector at the destination is $\mathbf{y}_{\mathrm{R}_{\mathrm{i}} \mathrm{D}}=\sqrt{\mathcal{E}_{\mathrm{R}}} \mathbf{g}_{i}^{\max } \alpha\left(\mathbf{h}_{i}^{\max }\right)^{\dagger} \mathbf{y}_{\mathrm{SR}_{\mathrm{i}}}+\mathbf{n}_{\mathrm{D}}$, where $\mathbf{n}_{\mathrm{D}}$ is the $N_{\mathrm{D}} \times 1$ AWGN vector satisfying $\mathbf{E}\left[\mathbf{n}_{\mathrm{D}} \mathbf{n}_{\mathrm{D}}^{\dagger}\right]=\mathbf{I}_{N_{\mathrm{D}}} \mathcal{N}_{0}$, and $\left\|\mathbf{g}_{i}^{\max }\right\|=\max _{1 \leq j \leq N_{\mathrm{R}}}\left\{\left\|\mathbf{g}_{i}^{j}\right\|\right\}$ denotes the largest channel vector between the $i$-th relay and the destination. The end-toend SNR is given by

$$
\gamma_{\mathrm{TAS} / \mathrm{MRC}}=\max _{1 \leq i \leq L}\left(\frac{X_{h_{i}} X_{g_{i}}}{X_{h_{i}}+X_{g_{i}}+1}\right),
$$

where $X_{h_{i}}=\frac{\mathcal{E}_{S}}{\mathcal{N}_{0}}\left\|\mathbf{h}_{i}^{\max }\right\|^{2}$ is the instantaneous SNR in the $i$-th source-to-relay link and $X_{g_{i}}=\frac{\mathcal{E}_{\mathrm{R}}}{\mathcal{N}_{0}}\left\|\mathbf{g}_{i}^{\max }\right\|^{2}$ is the instantaneous SNR in the $i$-th relay-to-destination link.

\section{B. TAS/SC Relaying}

In the source-to-relay link, a single antenna from $N_{\mathrm{S}}$ antennas at the source and a single antenna from $N_{\mathrm{R}}$ antennas at the $i$-th relay are jointly selected to maximize the received SNR at the relay. We denote $h_{i}^{j, k}$ as the channel coefficient from the $j$-th transmit antenna at the source to the $k$-th receive antenna at the relay. The received signal at the relay is $y_{\mathrm{SR}_{\mathrm{i}}}=\sqrt{\mathcal{E}_{\mathrm{S}}} h_{i}^{\max } x+n_{\mathrm{R}}$, where $n_{\mathrm{R}}$ is the AWGN with $\mathbf{E}\left[\left|n_{\mathrm{R}}\right|^{2}\right]=\mathcal{N}_{0}$ and $\left|h_{i}^{\max }\right|=\max _{1 \leq j \leq N_{\mathrm{S}}, 1 \leq k \leq N_{\mathrm{R}}}\left\{\left|h_{i}^{j, k}\right|\right\}$ denotes the largest channel coefficient between the source and the $i$-th relay.

In the relay-to-destination link, a single antenna from $N_{\mathrm{R}}$ antennas at the $i$-th relay and a single antenna from $N_{\mathrm{D}}$ antennas at the destination are jointly selected to maximize the received SNR at the destination. In this protocol, the relay applies a variable gain of $\alpha=1 / \sqrt{\mathcal{E}_{\mathrm{S}}\left|h_{i}^{\max }\right|^{2}+\mathcal{N}_{0}}$. We denote $g_{i}^{j, k}$ as the channel coefficient from the $j$-th transmit antenna at the relay to the $k$-th receive antenna at the destination. The received signal vector at the destination is $y_{\mathrm{R}_{\mathrm{i}} \mathrm{D}}=\sqrt{\mathcal{E}_{\mathrm{R}}} g_{i}^{\max } \alpha y_{\mathrm{SR}_{\mathrm{i}}}+n_{\mathrm{D}}$, where $n_{\mathrm{D}}$ is the AWGN with $\mathbf{E}\left[\left|n_{\mathrm{D}}\right|^{2}\right]=\mathcal{N}_{0}$, and $\left|g_{i}^{\max }\right|=\max _{1 \leq j \leq N_{\mathrm{R}}, 1 \leq k \leq N_{\mathrm{D}}}\left\{\left|g_{i}^{j, k}\right|\right\}$ denotes the largest channel coefficient between the $i$-th relay and the destination. The end-to-end SNR is given by

$$
\gamma_{\mathrm{TAS} / \mathrm{SC}}=\max _{1 \leq i \leq L}\left(\frac{Y_{h_{i}} Y_{g_{i}}}{Y_{h_{i}}+Y_{g_{i}}+1}\right),
$$

where $Y_{h_{i}}=\frac{\mathcal{E}_{\mathrm{S}}}{\mathcal{N}_{0}}\left|h_{i}^{\max }\right|^{2}$ is the instantaneous SNR in the $i$-th source-to-relay link and $Y_{g_{i}}=\frac{\mathcal{E}_{\mathrm{R}}}{\mathcal{N}_{0}}\left|g_{i}^{\max }\right|^{2}$ is the instantaneous SNR in the $i$-th relay-to-destination link.

\section{Preliminary Statistics}

We begin by evaluating the statistics of the SNR of single hop TAS/MRC and TAS/SC with $N_{t}$ transmit antennas and $N_{r}$ receive antennas. We consider a similar approach to [15] by expressing the first order expansion of the probability density function (pdf) of $\gamma_{k}=\frac{\mathcal{E}_{\mathrm{S}}}{\mathcal{N}_{0}}\left|h^{j, k}\right|^{2}$ around the origin $\left(\gamma \rightarrow 0^{+}\right)$ as

$$
f_{\gamma_{k}}^{\infty}(\gamma)=a_{k} \gamma^{\tau_{k}}+o\left(\gamma^{\tau_{k}}\right), \quad 0 \leq k \leq N_{r}
$$

where $a_{k}$ and $\tau_{k}$ are positive constants. We denote $h^{j, k}$ as the fading coefficient between the $j$-th antenna at the transmitter and the $k$-th antenna at the receiver. 
This article has been accepted for publication in a future issue of this journal, but has not been fully edited. Content may change prior to final publication.

TABLE I

SingLE Hop MiMO: Asymptotic STATistics of TAS/MRC AND TAS/SC

\begin{tabular}{|c|c|c|}
\hline Fading & Exact and Asymptotic PDF of $\gamma_{k}$ & $\lambda, \psi_{\mathrm{TAS} / \mathrm{MRC}}$, and $\phi_{\mathrm{TAS} / \mathrm{SC}}$ \\
\hline Rician & $\begin{array}{l}f_{\gamma_{k}}(\gamma)=\frac{n^{2}+1}{\bar{\gamma}_{k}} \exp \left(-n^{2}-\frac{\left(n^{2}+1\right) \gamma}{\bar{\gamma}_{k}}\right) I_{0}\left(2 n \sqrt{\frac{\left(n^{2}+1\right) \gamma}{\bar{\gamma}_{k}}}\right) \\
f_{\gamma_{k}}^{\infty}(\gamma)=\frac{n^{2}+1}{e^{n^{2}} \bar{\gamma}_{k}}+o(1)\end{array}$ & $\begin{array}{l}\lambda=N_{\mathrm{t}} N_{\mathrm{r}}, \psi_{\mathrm{TAS} / \mathrm{MRC}}=\frac{\left(n^{2}+1\right)^{N_{\mathrm{t}} N_{\mathrm{r}}}}{\left(N_{\mathrm{r}} !\right)^{N_{\mathrm{t}}} e^{n^{2} N_{\mathrm{t}} N_{\mathrm{r}}}} \\
\phi_{\mathrm{TAS} / \mathrm{SC}}=\frac{\left(n^{2}+1\right)^{N_{\mathrm{t}} N_{\mathrm{r}}}}{e^{n^{2} N_{\mathrm{t}} N_{\mathrm{r}}}}\end{array}$ \\
\hline Nakagami- $m$ & $\begin{array}{l}f_{\gamma_{k}}(\gamma)=\frac{m^{m} \gamma^{m-1}}{\bar{\gamma}_{k}^{m} \Gamma(m)} \exp \left(-\frac{m \gamma}{\bar{\gamma}_{k}}\right) \\
f_{\gamma_{k}}^{\infty}(\gamma)=\frac{m^{m} \gamma^{m-1}}{\Gamma(m) \bar{\gamma}_{k}^{m}}+o\left(\gamma^{m-1}\right)\end{array}$ & $\begin{array}{l}\lambda=m N_{\mathrm{t}} N_{\mathrm{r}}, \psi_{\mathrm{TAS} / \mathrm{MRC}}=\frac{m^{m N_{\mathrm{t}} N_{\mathrm{r}}}}{\left(\Gamma\left(m N_{\mathrm{r}}+1\right)\right)^{N_{\mathrm{t}}}} \\
\phi_{\mathrm{TAS} / \mathrm{SC}}=\frac{m^{m N_{\mathrm{t}} N_{\mathrm{r}}}}{(\Gamma(m+1))^{N_{\mathrm{t}} N_{\mathrm{r}}}}\end{array}$ \\
\hline Weibull & 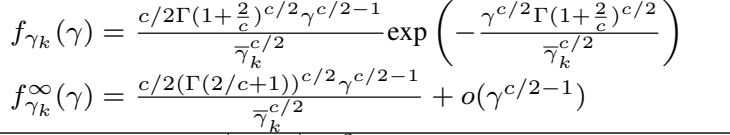 & $\begin{array}{l}\lambda=c N_{\mathrm{t}} N_{\mathrm{r}} / 2, \psi_{\mathrm{TAS} / \mathrm{MRC}}=\frac{(\Gamma(c / 2+1))^{N_{\mathrm{t}} N_{\mathrm{r}}}(\Gamma(2 / c+1))^{N_{\mathrm{t}} N_{\mathrm{r}} c / 2}}{\left(\Gamma\left(N_{\mathrm{r}} c / 2+1\right)\right)^{N_{\mathrm{t}}}} \\
\phi_{\mathrm{TAS} / \mathrm{SC}}=(\Gamma(2 / c+1))^{N_{\mathrm{t}} N_{\mathrm{r}} c / 2}\end{array}$ \\
\hline Generalized- $\mathcal{K}$ & 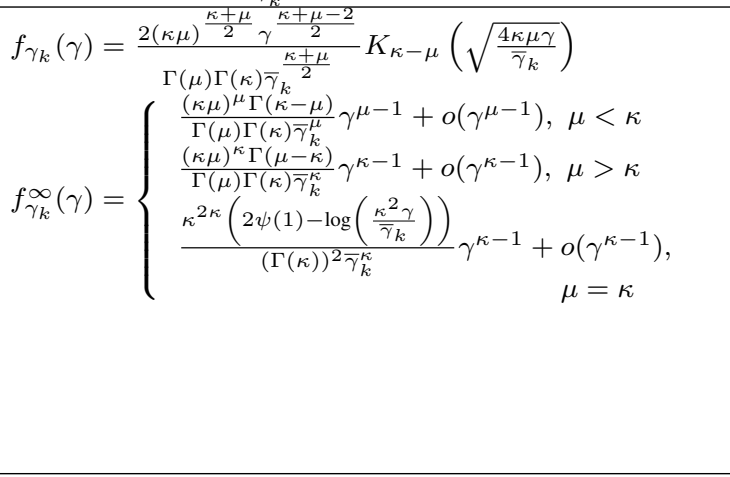 & 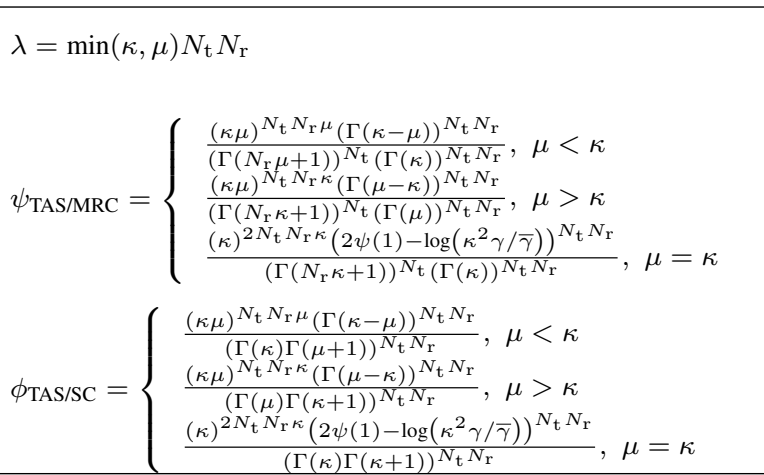 \\
\hline
\end{tabular}

\section{A. Single Hop TAS/MRC}

We denote $\mathbf{h}^{j}=\left[h^{j, 1}, \ldots, h^{j, N_{r}}\right], j \in\left\{1, \ldots, N_{t}\right\}$, as the channel vector between the $j$-th antenna at the transmitter and the $N_{r}$ antennas at the receiver. The instantaneous received SNR is $X_{\mathrm{TAS} / \mathrm{MRC}}=\frac{\mathcal{E}_{\mathrm{S}}}{\mathcal{N}_{0}}\left\|\mathbf{h}^{\text {max }}\right\|^{2}$ where $\left\|\mathbf{h}^{\text {max }}\right\|=$ $\max _{1 \leq j \leq N_{\mathrm{t}}}\left\{\left\|\mathbf{h}^{j}\right\|\right\}$ is the largest channel vector.

Proposition 1: The first order expansion of the cdf of $X_{\mathrm{TAS} / \mathrm{MRC}}$ as $\bar{\gamma}_{k}=\mathbf{E}\left[\frac{\mathcal{E}_{\mathrm{S}}}{\mathcal{N}_{0}}\left|h^{j, k}\right|^{2}\right] \rightarrow \infty$ can be expressed as

$$
F_{X_{\mathrm{TAS} / \mathrm{MRC}}}^{\infty}(\gamma)=\psi_{\mathrm{TAS} / \mathrm{MRC}}\left(\frac{\gamma}{\bar{\gamma}_{k}}\right)^{\lambda}+o\left(\bar{\gamma}_{k}^{-\lambda}\right),
$$

where $\psi_{\text {TAS/MRC }}$ and $\lambda$ for a range of fading conditions are detailed in Table I. In the sequel, we build on $\psi_{\text {TAS } / M R C}$ and $\lambda$ as the unifying parameters that describe the asymptotic behavior of TAS/MRC relaying. We note that the asymptotic results for single hop TAS/MRC in Nakagami- $m$ fading was previously presented in [15] which we include in Table I for completeness.

Proof: We apply Lemma 1 from [16] to derive the first order expansion of the cdf of $X_{\mathrm{MRC}}=\sum_{i=k}^{N_{r}} \gamma_{k}$ based on the pdf of $\gamma_{k}$ in (3), which results in

$$
F_{X_{\mathrm{MRC}}}^{\infty}(\gamma)=\frac{\prod_{k=1}^{N_{r}} a_{k} \Gamma\left(\tau_{k}+1\right) \gamma^{N_{r}\left(\tau_{k}+1\right)}}{N_{r}\left(\tau_{k}+1\right) \Gamma\left(N_{r}\left(\tau_{k}+1\right)\right)}+o\left(\bar{\gamma}^{-N_{r}\left(\tau_{k}+1\right)}\right) .
$$

Using (5), the first order expansion of the cdf of $X_{\mathrm{TAS} / \mathrm{MRC}}=$ $\frac{\mathcal{E}_{S}}{\mathcal{N}_{0}}\left\|\mathbf{h}^{\max }\right\|^{2}$ is calculated as

$F_{X_{\text {TASMRC }}}^{\infty}(\gamma)=\left(F_{X_{\mathrm{MRC}}}(\gamma)\right)^{N_{\mathrm{t}}}$

$$
=\frac{\left(\prod_{k=1}^{N_{r}} a_{k} \Gamma\left(\tau_{k}+1\right)\right)^{N_{t}} \gamma^{N_{t} N_{r}\left(\tau_{k}+1\right)}}{\left(N_{r}\left(\tau_{k}+1\right)\right)^{N_{t}}\left(\Gamma\left(N_{r}\left(\tau_{k}+1\right)\right)\right)^{N_{t}}}+o\left(\bar{\gamma}^{-N_{t} N_{r}\left(\tau_{k}+1\right)}\right) .
$$

Based on (6), the unifying parameters in (4) are derived as $\psi_{\mathrm{TAS} / \mathrm{MRC}}=\frac{\left(\prod_{k=1}^{N_{r}} a_{k} \Gamma\left(\tau_{k}+1\right)\right)^{N_{t}} \bar{\gamma}^{N_{t} N_{r}\left(\tau_{k}+1\right)}}{\left(N_{r}\left(\tau_{k}+1\right)\right)^{N_{t}}\left(\Gamma\left(N_{r}\left(\tau_{k}+1\right)\right)\right)^{N_{t}}}$ and $\lambda=$ $N_{\mathrm{t}} N_{\mathrm{r}}\left(\tau_{k}+1\right)$.

\section{B. Single Hop TAS/SC}

The instantaneous received SNR is $X_{\mathrm{TAS} / \mathrm{SC}}=\frac{\mathcal{E}_{\mathrm{S}}}{\mathcal{N}_{0}}\left|h^{\max }\right|^{2}$ where $\left|h^{\max }\right|=\max _{1 \leq j \leq N_{\mathrm{t}}, 1 \leq k \leq N_{r}}\left\{\left|h^{j, k}\right|\right\}$ is the largest channel coefficient between the transmitter and the receiver.

Proposition 2: The first order expansion of the cdf of $X_{\mathrm{TAS} / \mathrm{SC}}$ as $\bar{\gamma}_{k} \rightarrow \infty$ can be written as

$$
F_{X_{\mathrm{TAS} / \mathrm{SC}}}^{\infty}(\gamma)=\phi_{\mathrm{TAS} / \mathrm{SC}}\left(\frac{\gamma}{\bar{\gamma}_{k}}\right)^{\lambda}+o\left(\bar{\gamma}_{k}^{-\lambda}\right),
$$

where $\phi_{\mathrm{TAS} / \mathrm{SC}}$ and $\lambda$ are defined in Table I. In the sequel, we build on $\phi_{\mathrm{TAS} / \mathrm{SC}}$ and $\lambda$ as the unifying parameters that describe the asymptotic behavior of TAS/SC relaying.

Proof: We begin by deriving the first order expansion of the cdf of $\gamma_{k}$ given by

$$
F_{\gamma_{k}}^{\infty}(\gamma)=\int_{0}^{\infty} f_{\gamma_{k}}^{\infty}(\gamma) d \gamma=\frac{a_{k} \gamma^{\tau_{k}+1}}{\tau_{k}+1}+o\left(\bar{\gamma}^{-\tau_{k}-1}\right)
$$

Using (8), the first order expansion of the cdf of $X_{\mathrm{TAS} / \mathrm{SC}}=$ $\frac{\mathcal{E}_{\mathrm{S}}}{\mathcal{N}_{0}}\left|h^{\max }\right|^{2}$ is given by

$$
\begin{aligned}
F_{X_{\text {TAS } / S C}}^{\infty}(\gamma) & =\left(F_{\gamma_{k}}(\gamma)\right)^{N_{\mathrm{t}} N_{\mathrm{r}}} \\
& =\frac{a_{k}^{N_{\mathrm{t}} N_{\mathrm{r}}} \gamma^{N_{\mathrm{t}} N_{\mathrm{r}}\left(\tau_{k}+1\right)}}{\left(\tau_{k}+1\right)^{N_{\mathrm{t}} N_{\mathrm{r}}}}+o\left(\bar{\gamma}^{-N_{\mathrm{t}} N_{\mathrm{r}}\left(\tau_{k}+1\right)}\right) .
\end{aligned}
$$


This article has been accepted for publication in a future issue of this journal, but has not been fully edited. Content may change prior to final publication.

TABLE II

MiMO Multi-RElay Networks: Asymptotic Statistics of TAS/MRC and TAS/SC

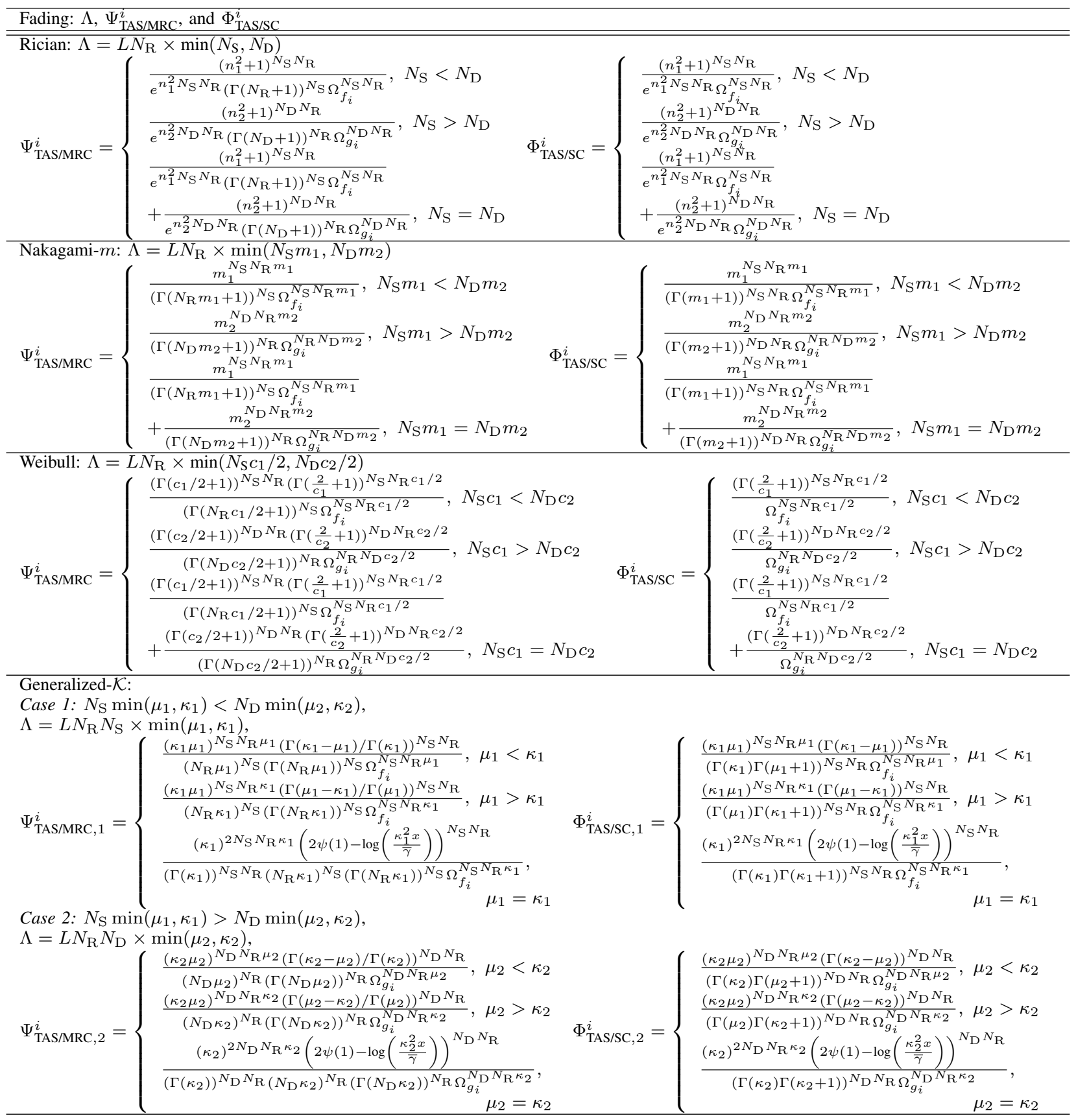

Based on (9), the unifying parameters in (7) are derived as $\phi_{\mathrm{TAS} / \mathrm{SC}}=\frac{a_{k}^{N_{\mathrm{t}} N_{\mathrm{r}}} \bar{\gamma}^{N_{\mathrm{t}} N_{\mathrm{r}}\left(\tau_{k}+1\right)}}{\left(\tau_{k}+1\right)^{N_{\mathrm{t}} N_{\mathrm{r}}}}$ and $\lambda=N_{\mathrm{t}} N_{\mathrm{r}}\left(\tau_{k}+1\right)$.

\section{Outage Probability}

In this section, we derive new closed-form asymptotic expressions for the outage probability of MIMO multi-relay networks with TAS/MRC and TAS/SC in Rician, Nakagami$m$, Weibull, and Generalized- $\mathcal{K}$ fading. The asymptotic outage probability is the probability that the end-to-end SNRs $\gamma_{\mathrm{TAS} / \mathrm{MRC}}$ in (1) and $\gamma_{\mathrm{TAS} / \mathrm{SC}}$ in (2) fall below a given SNR threshold $\gamma_{\text {th }}$ according to $P_{\text {out }}^{\infty}=\operatorname{Pr}\left\{\gamma_{\text {eq }} \leq \gamma_{\text {th }}\right\}=F_{\gamma_{\text {eq }}}^{\infty}\left(\gamma_{\text {th }}\right)$, where $F_{\gamma_{\text {eq }}}^{\infty}(\cdot)$ is the first order expansion of the cdf of

\section{$\gamma_{\mathrm{eq}} \in\left\{\gamma_{\mathrm{TAS} / \mathrm{MRC}}, \gamma_{\mathrm{TAS} / \mathrm{SC}}\right\}$}

We consider a similar approach to [17] in which $F_{\gamma_{\mathrm{eq}}}^{\infty}(\cdot)$ is derived based on the first order expansions of the cdf's for each hop. As such, we can apply the results for single hop TAS/MRC in (4) and TAS/SC in (7) to derive the asymptotic outage probability for MIMO multi-relay networks. We consider that all the source-to-relay and relay-to-destination channel gains are mutually independent. In the following, we express the per-hop average received SNRs in terms of a reference SNR $\bar{\gamma}$. Accordingly, we define $\bar{\gamma}_{f_{i}}=\Omega_{f_{i}} \bar{\gamma}$, where $\bar{\gamma}_{f_{i}}=\frac{\mathcal{E}_{S}}{\mathcal{N}_{0}}$ is the average SNR between each antenna pair in the source-to-relay link and $\Omega_{f_{i}}$ is a positive constant. We 
This article has been accepted for publication in a future issue of this journal, but has not been fully edited. Content may change prior to final publication.

also define $\bar{\gamma}_{g_{i}}=\Omega_{g_{i}} \bar{\gamma}$, where $\bar{\gamma}_{g_{i}}=\frac{\mathcal{E}_{\mathrm{R}}}{\mathcal{N}_{0}}$ is the average SNR between each antenna pair in the relay-to-destination link and $\Omega_{g_{i}}$ is a positive constant.

\section{A. TAS/MRC Relaying}

Based on the asymptotic CDF parameters $\psi_{\mathrm{TAS} / \mathrm{MRC}}$ and $\lambda$ in Table I, the first order expansion of $F_{\gamma_{\mathrm{TAS} / \mathrm{MRC}}}(\gamma)$ as $\gamma \rightarrow 0^{+}$ is evaluated for the three separable cases of $N_{\mathrm{S}}$ relative to $N_{\mathrm{D}}$. We present the first order expansion of the cdf of $\gamma_{\mathrm{TAS} / \mathrm{MRC}}$ as

$$
F_{\gamma_{\mathrm{TAS} / \mathrm{MRC}}}^{\infty}(\gamma)=\prod_{i=1}^{L} \Psi_{\mathrm{TAS} / \mathrm{MRC}}^{i}\left(\frac{\gamma}{\bar{\gamma}}\right)^{\Lambda}+o\left(\bar{\gamma}^{-\Lambda}\right)
$$

where $\Lambda$ and $\Psi_{\text {TAS/MRC }}^{i}$ are defined according to the fading channels in Table II.

\section{B. TAS/SC Relaying}

The first order expansion of the cdf of $\gamma_{\mathrm{TAS} / \mathrm{SC}}$ is calculated using $\phi_{\mathrm{TAS} / \mathrm{SC}}$ and $\lambda$ in Table I for the three separable cases of $N_{\mathrm{S}}$ relative to $N_{\mathrm{D}}$. The cdf's of $\gamma_{\mathrm{TAS} / \mathrm{SC}}$ is presented as

$$
F_{\gamma_{\mathrm{TAS} / \mathrm{SC}}}^{\infty}(\gamma)=\prod_{i=1}^{L} \Phi_{\mathrm{TAS} / \mathrm{SC}}^{i}\left(\frac{\gamma}{\bar{\gamma}}\right)^{\Lambda}+o\left(\bar{\gamma}^{-\Lambda}\right)
$$

where $\Lambda$ and $\Phi_{\mathrm{TAS} / \mathrm{SC}}^{i}$ are defined according to the fading channels given in Table II. Comparing (10) and (11), we note that the main difference lies in the definition of the asymptotic parameters $\Psi_{\mathrm{TAS} / \mathrm{MRC}}^{i}$ and $\Phi_{\mathrm{TAS} / \mathrm{SC}}^{i}$.

\section{Symbol ERror Rate}

In this section, we derive the asymptotic SER of TAS/MRC and TAS/SC relaying with the higher order constellations of $M$-PSK and $M$-QAM. To obtain further insights, we present a fundamental comparison of TAS/MRC and TAS/SC relaying based on their array gains in Rician, Nakagami- $m$, Weibull, and Generalized- $\mathcal{K}$ fading.

The asymptotic SER with M-PSK can be evaluated using [18]

$$
P_{e}^{\infty}=\frac{a_{\mathrm{MPSK}}}{\pi} \int_{0}^{\Theta} \int_{0}^{\infty} \frac{e^{-\gamma \frac{a_{\mathrm{MPSK}}}{\sin ^{2} \theta}}}{\sin ^{2} \theta} F_{\gamma}^{\infty}(\gamma) d \gamma d \theta
$$

where $F_{\gamma}(\gamma)$ is the cdf of the end-to-end SNR, $\Theta=\pi(\mathcal{M}-$ 1) $/ \mathcal{M}, a_{\mathrm{MPSK}}=\sin ^{2}(\pi / \mathcal{M})$, and $\mathcal{M}$ represents the constellation size. For TAS/MRC relaying, substituting (10) into (12) and solving the nested integrals results in

$$
\begin{aligned}
& P_{e, \mathrm{TAS} / \mathrm{MRC}}^{\infty}=\left(\frac{\Gamma(1 / 2 \Lambda)}{2 \sqrt{\pi}}+\frac{\Gamma(1+\Lambda) \cos \left(\frac{\pi}{\mathcal{M}}\right)}{\pi}\right. \\
& \left.\times{ }_{2} F_{1}\left(\frac{1}{2}, \frac{1}{2}-\Lambda ; \frac{3}{2} ; \cos ^{2}\left(\frac{\pi}{\mathcal{M}}\right)\right)\right) \\
& \times \prod_{i=1}^{L} \Psi_{\mathrm{TAS} / \mathrm{MRC}}^{i}\left(a_{\mathrm{MPSK}} \bar{\gamma}\right)^{-\Lambda}+o\left(\bar{\gamma}^{-\Lambda}\right)
\end{aligned}
$$

with ${ }_{2} F_{1}(a, b ; c ; z)$ symbolizing the Gauss hypergeometric function [19, eq. (9.100)]. For TAS/SC relaying, the asymp-

\begin{tabular}{|c|c|}
\hline Fading & Array gain ratio, $\frac{\mathcal{G}_{A, \mathrm{TAS} / \mathrm{MRC}}}{\mathcal{G}_{A, \mathrm{TAS} / \mathrm{SC}}}$ \\
\hline Rician & $\begin{array}{l}\frac{\mathcal{G}_{A, \mathrm{TAS} / \mathrm{MRC}}}{\mathcal{G}_{A, \mathrm{TAS} / \mathrm{SC}}} \\
=\left\{\begin{array}{l}\left(\Gamma\left(N_{\mathrm{R}}+1\right)\right)^{\frac{1}{N_{\mathrm{R}}}}, N_{\mathrm{S}}<N_{\mathrm{D}} \\
\left(\Gamma\left(N_{\mathrm{D}}+1\right)\right)^{\frac{1}{N_{\mathrm{D}}}}, N_{\mathrm{S}}>N_{\mathrm{D}}\end{array}\right.\end{array}$ \\
\hline Nakagami- $m$ & $\begin{array}{l}\frac{\mathcal{G}_{A, \mathrm{TAS} / \mathrm{MRC}}}{\mathcal{G}_{A, \mathrm{TAS} / \mathrm{SC}}} \\
=\left\{\begin{array}{l}\left(\frac{\left(\Gamma\left(N_{\mathrm{R}} m_{1}+1\right)\right)^{N_{\mathrm{S}}}}{\left(\Gamma\left(m_{1}+1\right)\right)^{N_{\mathrm{S}} N_{\mathrm{R}}}}\right)^{\frac{1}{N_{\mathrm{R}} m_{1}}}, N_{\mathrm{S}} m_{1}<N_{\mathrm{D}} m_{2} \\
\left(\frac{\left(\Gamma\left(N_{\mathrm{D}} m_{2}+1\right)\right)^{N_{\mathrm{R}}}}{\left(\Gamma\left(m_{2}+1\right)\right)^{N_{\mathrm{D}} N_{\mathrm{R}}}}\right)^{\frac{1}{N_{\mathrm{D}} m_{2}}}, N_{\mathrm{S}} m_{1}>N_{\mathrm{D}} m_{2}\end{array}\right.\end{array}$ \\
\hline Weibull & $\begin{array}{l}\frac{\mathcal{G}_{A, \mathrm{TAS} / \mathrm{MRC}}}{\mathcal{G}_{A, \mathrm{TAS} / \mathrm{SC}}} \\
=\left\{\begin{array}{l}\left(\frac{\Gamma\left(N_{\mathrm{R}} c_{1} / 2+1\right)}{\left(\Gamma\left(c_{1} / 2+1\right)\right)^{N_{\mathrm{R}}}}\right)^{\frac{1}{N_{\mathrm{R}} c_{1} / 2}}, N_{\mathrm{S}} c_{1}<N_{\mathrm{D}} c_{2} \\
\left(\frac{\Gamma\left(N_{\mathrm{D}} c_{2} / 2+1\right)}{\left(\Gamma\left(c_{2} / 2+1\right)\right)^{N_{\mathrm{D}}}}\right)^{\frac{1}{N_{\mathrm{D}} c_{2} / 2}}, N_{\mathrm{S}} c_{1}>N_{\mathrm{D}} c_{2}\end{array}\right.\end{array}$ \\
\hline Generalized- $\mathcal{K}$ & $\begin{array}{l}\text { Case 1: } N_{\mathrm{S}} \min \left(\mu_{1}, \kappa_{1}\right)<N_{\mathrm{D}} \min \left(\mu_{2}, \kappa_{2}\right), \\
\frac{\mathcal{G}_{A, \mathrm{TAS} / \mathrm{MRC}}}{\mathcal{G}_{A, \mathrm{TAS} / \mathrm{SC}}}=\left\{\begin{array}{c}\left(\frac{\left(\Gamma\left(N_{\mathrm{R}} \mu_{1}+1\right)\right)}{\left(\Gamma\left(\mu_{1}+1\right)\right)^{N_{\mathrm{R}}}}\right)^{\frac{1}{N_{\mathrm{R}} \mu_{1}}}, \\
\mu_{1}<\kappa_{1} \\
\left(\frac{\left(\Gamma\left(N_{\mathrm{R}} \kappa_{1}+1\right)\right)}{\left(\Gamma\left(\kappa_{1}+1\right)\right)^{N_{\mathrm{R}}}}\right)^{\frac{1}{N_{\mathrm{R}} \kappa_{1}}} \\
\mu_{1}>\kappa_{1}\end{array}\right. \\
\text { Case 2: } N_{\mathrm{S}} \min \left(\mu_{1}, \kappa_{1}\right)>N_{\mathrm{D}} \min \left(\mu_{2}, \kappa_{2}\right), \\
\frac{\mathcal{G}_{A, \mathrm{TAS} / \mathrm{MRC}}}{\mathcal{G}_{A, \mathrm{TAS} / \mathrm{SC}}}=\left\{\begin{array}{c}\left(\frac{\left(\Gamma\left(N_{\mathrm{D}} \mu_{2}+1\right)\right)}{\left(\Gamma\left(\mu_{2}+1\right)\right)^{N_{\mathrm{D}}}}\right) \begin{array}{c}\frac{1}{N_{\mathrm{D}} \mu_{2}} \\
\mu_{2}<\kappa_{2}\end{array} \\
\left(\frac{\left(\Gamma\left(N_{\mathrm{D}} \kappa_{2}+1\right)\right)}{\left.\left(\Gamma\left(\kappa_{2}+1\right)\right)^{N_{\mathrm{D}}}\right)}\right)^{\frac{1}{N_{\mathrm{D}} \kappa_{2}}}, \\
\mu_{2}>\kappa_{2}\end{array}\right.\end{array}$ \\
\hline
\end{tabular}
totic SER with M-PSK is obtained by replacing $\Psi_{\mathrm{T}}^{i}$ in (13) with $\Phi_{\mathrm{TAS} / \mathrm{SC}}^{i}$.
TABLE III

MIMO Multi-RELAY NETWORKS: ARRAy GAIN RATIOS OF TAS/MRC AND TAS/SC

The asymptotic SER with $M$-QAM is evaluated using [18]

$$
\begin{aligned}
& P_{e}^{\infty}=\frac{6\left(1-\frac{1}{\sqrt{\mathcal{M}}}\right)}{\pi(\mathcal{M}-1)} \int_{0}^{\frac{\pi}{2}} \int_{0}^{\infty} \frac{e^{-\gamma \frac{a_{\mathrm{MQAM}}}{\sin ^{2} \theta}}}{\sin ^{2} \theta} F_{\gamma}^{\infty}(\gamma) d \gamma d \theta \\
& -\frac{6\left(1-\frac{1}{\sqrt{\mathcal{M}}}\right)^{2}}{\pi(\mathcal{M}-1)} \int_{0}^{\frac{\pi}{4}} \int_{0}^{\infty} \frac{e^{-\gamma \frac{a_{\mathrm{MQAM}}}{\sin ^{2} \theta}}}{\sin ^{2} \theta} F_{\gamma}^{\infty}(\gamma) d \gamma d \theta,
\end{aligned}
$$

where $a_{\mathrm{MQAM}}=3 / 2(\mathcal{M}-1)$. For TAS/MRC relaying, substituting (10) into (14) and solving the nested integrals results in

$$
\begin{aligned}
& P_{e, \mathrm{TAS} / \mathrm{MRC}}^{\infty}=\left(\frac{2 \Gamma(1 / 2+\Lambda)}{\sqrt{\pi}}\left(1-\frac{1}{\sqrt{\mathcal{M}}}\right)\right. \\
& \left.-\frac{2 \Gamma(1+\Lambda)}{\pi}\left(1-\frac{1}{\sqrt{\mathcal{M}}}\right)^{2} B_{\frac{1}{2}}\left(\frac{1}{2}+\Lambda, \frac{1}{2}\right)\right) \\
& \times \prod_{i=1}^{L} \Psi_{\mathrm{TAS} / \mathrm{MRC}}^{i}\left(a_{\mathrm{MQAM}} \bar{\gamma}\right)^{-\Lambda}+o\left(\bar{\gamma}^{-\Lambda}\right)
\end{aligned}
$$

with $B_{z}(a, b)=\int_{0}^{z} t^{a-1}(1-t)^{b-1} d t$ symbolizing the incomplete beta function [19, eq. (8.391)]. For TAS/SC relaying, the asymptotic SER with M-QAM is found by replacing $\Psi_{\mathrm{TAS} / \mathrm{MRC}}^{i}$ in (15) with $\Phi_{\mathrm{TAS} / \mathrm{SC}}^{i}$. 


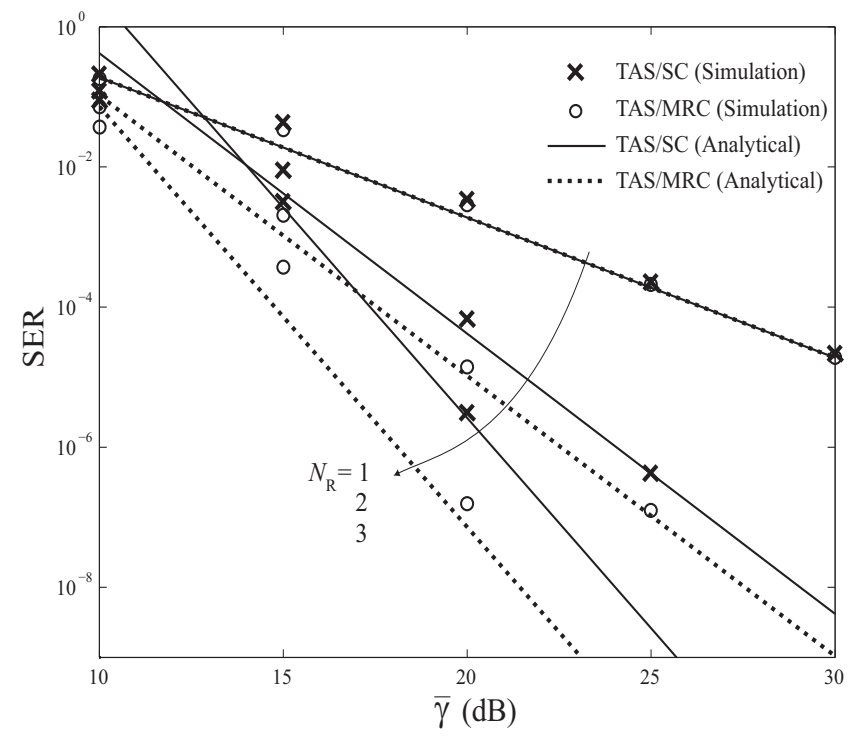

Fig. 1. SER with 8-PSK in Rician fading with $n_{1}=n_{2}=1, N_{\mathrm{S}}=1$, $N_{\mathrm{D}}=2$, and $L=2$.

\section{A. Comparison of TAS/SC and TAS/MRC}

To obtain further insights, our asymptotic solutions can be re-expressed as $P_{e}^{\infty}=\left(\mathcal{G}_{A} \bar{\gamma}\right)^{-\mathcal{G}_{D}}+o\left(\bar{\gamma}^{-\mathcal{G}_{D}}\right)$, where $\mathcal{G}_{D}$ is the diversity order, which determines the asymptotic slope of the SER curve, and $\mathcal{G}_{A}$ is the array gain, which represents the SNR advantage of the SER curve with respect to $\bar{\gamma}^{-\mathcal{G}_{D}}$. Based on (13) and (15), we see that the diversity order for TAS/MRC and TAS/SC is $\mathcal{G}_{D}=\Lambda$.

The SNR gap between TAS/MRC and TAS/SC can be concisely expressed as a ratio of their respective array gains. Based on (13) and (15), we find that the ratio is independent of the signal constellations and is derived as

$$
\frac{\mathcal{G}_{A, \mathrm{TAS} / \mathrm{MRC}}}{\mathcal{G}_{A, \mathrm{TAS} / \mathrm{SC}}}=\left(\frac{\prod_{i=1}^{L} \Phi_{\mathrm{TAS} / \mathrm{SC}}^{i}}{\prod_{i=1}^{L} \Psi_{\mathrm{TAS} / \mathrm{MRC}}^{i}}\right)^{\frac{1}{\mathcal{G}_{D}}} .
$$

Setting $\Phi_{\mathrm{TAS} / \mathrm{SC}}^{i}=\Phi_{\mathrm{TAS} / \mathrm{SC}}$ and $\Psi_{\mathrm{TAS} / \mathrm{MrC}}^{i}=\Psi_{\mathrm{TAS} / \mathrm{MRC}}$, $\forall i \in\{1, \ldots, L\}$, which corresponds to having equal per-hop SNRs (i.e., $\Omega_{f_{i}}=\Omega_{f}$ and $\Omega_{g_{i}}=\Omega_{g_{i}}, \forall i$ ), the ratio in (16) simplifies to

$$
\frac{\mathcal{G}_{A, \mathrm{TAS} / \mathrm{MRC}}}{\mathcal{G}_{A, \mathrm{TAS} / \mathrm{SC}}}=\left(\frac{\Phi_{\mathrm{TAS} / \mathrm{SC}}}{\Psi_{\mathrm{TAS} / \mathrm{MRC}}}\right)^{\frac{L}{\mathcal{G}_{D}}}
$$

We note that the diversity order of TAS/SC and TAS/MRC is defined in terms of the number of relays, $L$. As such, we find that the SNR gap between TAS/MRC and TAS/SC in (17) is independent of $L$ and only dependent on the number of antennas and the fading parameters. The array gain ratio in (17) is defined in Table III for Rician, Nakagami- $m$, Weibull, and Generalized- $\mathcal{K}$ fading with unbalanced fading conditions and unequal antenna configurations between the hops. It is interesting to note that our results may be extended to consider TAS with generalized selection combining (GSC) to bridge the SNR gap between TAS/MRC and TAS/SC. In TAS/GSC,

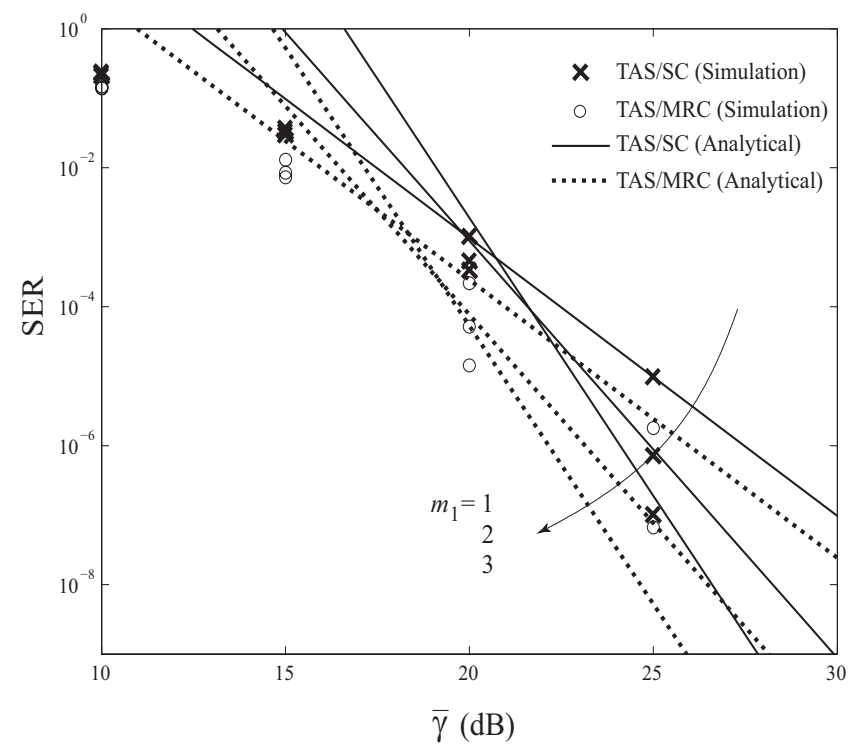

Fig. 2. SER with 16-QAM in Nakagami- $m$ fading with $m_{2}=1, N_{\mathrm{S}}=1$, $N_{\mathrm{R}}=2, N_{\mathrm{D}}=3$, and $L=2$.

a subset of antennas with the highest SNRs are activated at each receiver. As such, TAS/GSC offers a compelling costperformance tradeoff between TAS/MRC and TAS/SC.

\section{NUMERICAL EXAMPLES}

In this section, we validate the preceding analytical results and highlight the SER comparison between TAS/MRC and TAS/SC. In the plots, ' $x$ ' denotes the simulation points for TAS/SC and ' $O$ ' denotes the simulation points for TAS/MRC. The solid curves represent the asymptotic SER of TAS/SC, and the dashed curves represent the asymptotic SER of TAS/MRC. In all the plots, we see that the analytical curves accurately characterize the diversity order and the array gain for a wide range of antenna configurations and fading conditions.

Fig. 1 plots the SER of TAS/MRC and TAS/SC with 8PSK in Rician fading. The asymptotic SER is calculated using (13) with $\Psi_{\text {TAS/MRC }}^{i}$ in Table II for TAS/MRC, and $\Phi_{\mathrm{TAS} / \mathrm{SC}}^{i}$ in Table II for TAS/SC. We set $L=2, N_{\mathrm{S}}=1$, $N_{\mathrm{D}}=3, n_{1}=n_{2}=1$. Note that the diversity order of both TAS/MRC and TAS/SC increases with the number of antennas, $N_{\mathrm{R}}$, according to $\mathcal{G}_{D}=L N_{\mathrm{R}} \times \min \left(N_{\mathrm{S}}, N_{\mathrm{D}}\right)$. As expected, there is no SNR gap when $N_{\mathrm{R}}=1$ (i.e., $\left.\mathcal{G}_{A, \mathrm{TAS} / \mathrm{MRC}} / \mathcal{G}_{A, \mathrm{TAS} / \mathrm{SC}}=1\right)$. Furthermore, the SNR gap between TAS/MRC and TAS/SC increases with $N_{\mathrm{R}}$ according to the ratio in Table III.

Fig. 2 plots the SER of TAS/MRC and TAS/SC with 16-QAM in Nakagami- $m$ fading. The analytical curves for TAS/MRC are evaluated by substituting $\Psi_{\text {TAS/MRC }}^{i}$ in Table II into (15). The analytical curves for TAS/SC are based on (15) with $\Phi_{\text {TAS/SC }}^{i}$ in Table II. We set $L=2, N_{\mathrm{S}}=1, N_{\mathrm{R}}=2$, $N_{\mathrm{D}}=3$, and $m_{2}=1$. Note that the diversity order of both TAS/MRC and TAS/SC increases with the fading parameter $m_{1}$, according to $\mathcal{G}_{D}=L N_{\mathrm{R}} \times \min \left(N_{\mathrm{S}} m_{1}, N_{\mathrm{D}} m_{2}\right)$. As expected, there is no SNR gap when $N_{\mathrm{S}}=N_{\mathrm{R}}=N_{\mathrm{D}}=1$ 


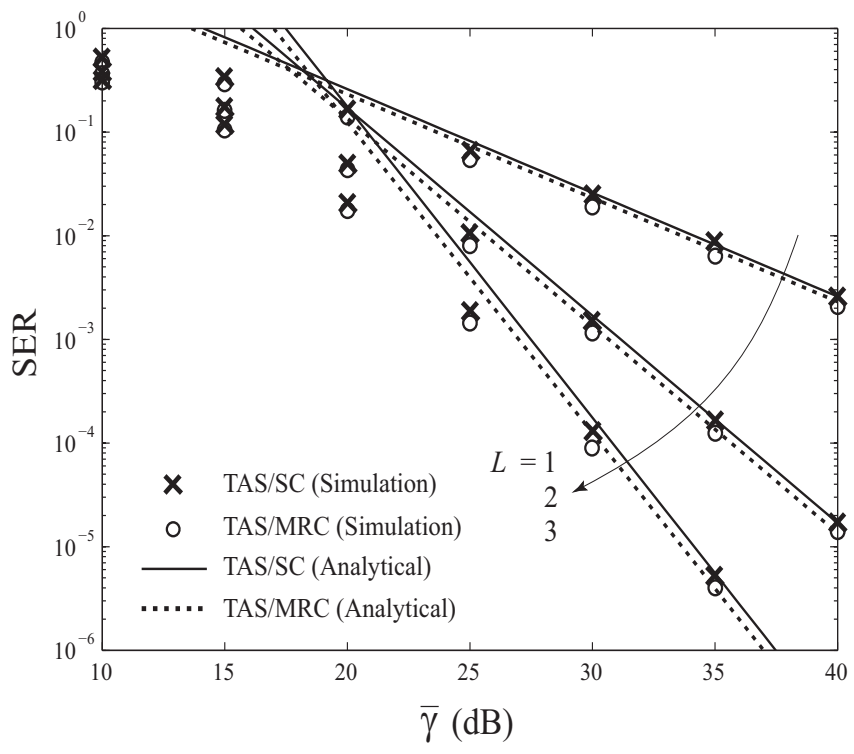

Fig. 3. SER with 16-QAM in Weibull fading with $c_{1}=c_{2}=1, N_{\mathrm{S}}=2$, $N_{\mathrm{R}}=1$, and $N_{\mathrm{D}}=2$.

(i.e., $\mathcal{G}_{A, \mathrm{TAS} / \mathrm{MRC}} / \mathcal{G}_{A, \mathrm{TAS} / \mathrm{SC}}=1$ ). Furthermore, the SNR gap between TAS/MRC and TAS/SC increases with $m_{1}$ as predicted in Table III.

Fig. 3 plots the SER with 16-QAM in Weibull fading using (15) and $\Psi_{\text {TAS/MRC }}^{i}$ in Table II for TAS/MRC, and $\Phi_{\text {TAS/SC }}^{i}$ in Table II for TAS/SC. We set $N_{\mathrm{S}}=2, N_{\mathrm{R}}=1, N_{\mathrm{D}}=2$, and $c_{1}=c_{2}=1$. Observe that the diversity order of both TAS/MRC and TAS/SC increases with the number of relays, $L$, according to $\mathcal{G}_{D}=L N_{\mathrm{R}} \times \min \left(N_{\mathrm{S}} c_{1} / 2, N_{\mathrm{D}} c_{2} / 2\right)$. It can also be seen that the SNR gap between TAS/MRC and TAS/SC is independent of $L$.

Fig. 4 plots the SER of TAS/MRC and TAS/SC with 8 -PSK in Generalized- $\mathcal{K}$ fading. The asymptotic SER for TAS/MRC is calculated using $\Psi_{\text {TAS/MRC }}^{i}$ in Table II and (13). The asymptotic SER for TAS/SC is evaluated using $\Phi_{\mathrm{TAS} / \mathrm{SC}}^{i}$ in Table II and (13). We set $N_{\mathrm{S}}=1, N_{\mathrm{R}}=2, N_{\mathrm{D}}=2, \mu_{1}=2$, $\mu_{2}=1, \kappa_{1}=3$, and $\kappa_{2}=1$. Observe that the diversity order of both TAS/MRC and TAS/SC increases with the number of relays, $L$. It can also be seen that the SNR gap between TAS/MRC and TAS/SC is independent of $L$.

\section{CONCLUSION}

We examined TAS/MRC and TAS/SC in MIMO multi-relay networks over Rician, Nakagami- $m$, Weibull, and Generalized$\mathcal{K}$ fading channels. New asymptotic expressions for the outage probability and the SER were derived for arbitrary $L$ two-hop relay links with $N_{\mathrm{S}}, N_{\mathrm{R}}$, and $N_{\mathrm{D}}$ antennas at the source, the relays, and the destination, respectively. Based on the asymptotic SER, we derived the diversity order and the array gain for $M$-PSK and $M$-QAM. Our results reveal that TAS/MRC and TAS/SC achieve the same diversity order. As such, the SNR gap between the two protocols is entirely dependent on the array gain. Motivated by this, we characterized the SNR gap as a simple ratio of their respective array gains. An interesting

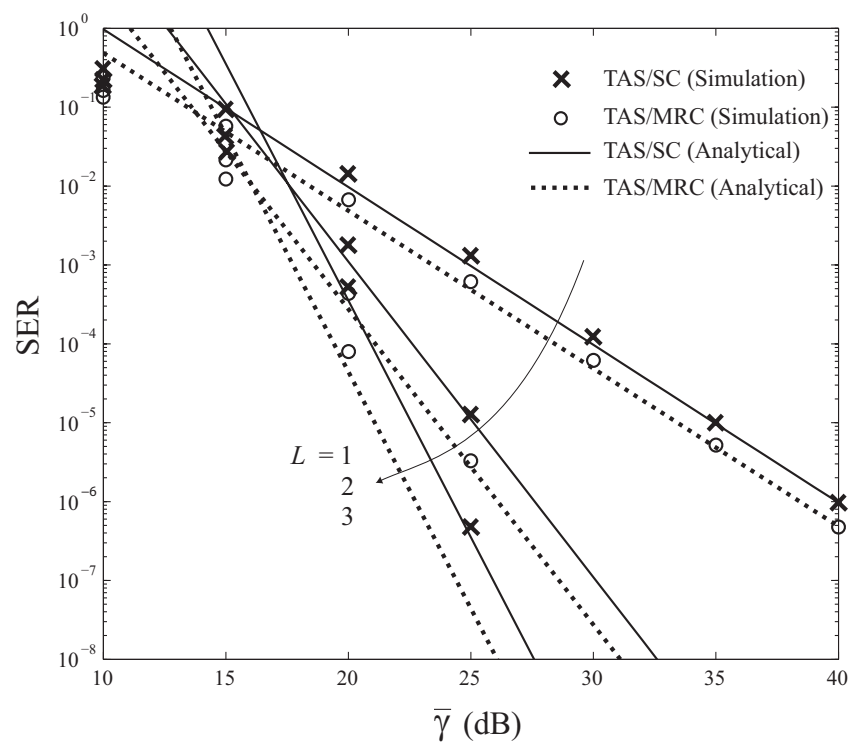

Fig. 4. SER with 8-PSK in Generalized- $\mathcal{K}$ fading with $\mu_{1}=2, \mu_{2}=1$, $\kappa_{1}=3, \kappa_{2}=1, N_{\mathrm{S}}=1, N_{\mathrm{R}}=2$, and $N_{\mathrm{D}}=2$.

observation is made that the SNR gap is constant regardless of $L$ for equal average received SNRs at the relays and the destination. We concisely express the SNR gap in terms of the number of antennas and the fading parameters for Rician, Nakagami- $m$, Weibull, and Generalized- $\mathcal{K}$ fading.

\section{REFERENCES}

[1] C. Hoymann, W. Chen, J. Montojo, A. Golitschek, C. Koutsimanis, and $\mathrm{X}$. Shen, "Relaying operation in 3GPP LTE: Challenges and solutions," IEEE Commun. Mag., vol. 50, pp. 156-162, Feb. 2012.

[2] A. Damnjanovic, J. Montojo, J. Cho, H. Ji, J. Yang, and P. Zong, "UE's role in LTE advanced heterogeneous networks," IEEE Commun. Mag., vol. 50, pp. 164-176, Feb. 2012.

[3] 3GPP TR 36.826 v0.10.0, "E-UTRA; Relay radio transmission and reception (Release 11)," Aug. 2011.

[4] Z. Zhou and B. Vucetic, "A cooperative beamforming scheme in MIMO relay broadcast channels," IEEE Trans. Wireless Commun., vol. 10, pp. 940-947, Mar. 2011.

[5] Z. Wang, W. Chen, and J. Li, "Efficient beamforming for MIMO relaying broadcast channel with imperfect channel estimation," IEEE Trans. Signal Process., vol. 61, pp. 419-426, Jan. 2012.

[6] P. L. Yeoh, M. Elkashlan, and I. B. Collings, "Exact and asymptotic SER of distributed TAS/MRC in MIMO relay networks," IEEE Trans. Wireless Commun.,, vol. 10, pp. 751-756, Mar. 2011.

[7] M. Elkashlan, P. Yeoh, N. Yang, T. Duong, and C. Leung, "A comparison of two MIMO relaying protocols in Nakagami- $m$ fading," IEEE Trans. Veh. Technol., accepted to appear.

[8] H. A. Suraweera, P. J. Smith, A. Nallanathan, and J. S. Thompson, "Amplify-and-forward relaying with optimal and suboptimal transmit antenna selection," IEEE Trans. Wireless Commun., vol. 10, pp. 18741885, Jun. 2011.

[9] H. Suraweera, G. K. Karagiannidis, Y. Li, H. K. Garg, A. Nallanathan, and B. Vucetic, "Amplify-and-forward relay transmission with end-toend antenna selection," in Proc. IEEE Wireless Commun. and Netw. Conf. (WCNC), Sydney, Australia, Apr. 2010, pp. 1-6.

[10] B. Maham and A. Hjorungnes, "Asymptotic performance analysis of amplify-and-forward cooperative networks in a Nakagami- $m$ fading environment," IEEE Commun. Lett., vol. 13, pp. 300-302, May 2009.

[11] - ,Performance analysis of amplify-and-forward opportunistic relaying in Rician fading," IEEE Signal Process. Lett., vol. 16, pp. 643646, Aug. 2009. 
[12] P. S. Bithas, N. C. Sagias, P. T. Mathiopoulos, G. K. Karagiannidis, and A. A. Rontogiannis, "General ergodic capacity bounds for fixed-gain AF dual-hop relaying systems," IEEE Trans. Veh. Technol., vol. 60, pp. 3814-3824, Oct. 2011.

[13] N. C. Sagias and G. K. Karagiannidis, "Gaussian class multivariate Weibull distributions: Theory and applications in fading channels," IEEE Trans. Inf. Theory, vol. 51, pp. 3608-3619, Oct. 2005.

[14] P. S. Bithas, N. C. Sagias, P. T. Mathiopoulos, G. K. Karagiannidis, and A. A. Rontogiannis, "On the performance analysis of digital communications over Generalized- $K$ fading channels," IEEE Commun. Lett., vol. 10, pp. 353-355, May 2006.

[15] Z. Chen, Z. Chi, Y. Li, and B. Vucetic, "Error performance of maximalratio combining with transmit antenna selection in flat Nakagami- $m$ fading channels," IEEE Trans. Wireless Commun., vol. 8, pp. 424-431,
Jan. 2009

[16] M. Elkashlan, P. L. Yeoh, R. H. Y. Louie, and I. B. Collings, "On the exact and asymptotic SER of receive diversity with multiple amplifyand-forward relays," IEEE Trans. Veh. Technol., vol. 59, pp. 4602-4608, Nov. 2010.

[17] Y. Li and S. Kishore, "Asymptotic analysis of amplify-and-forward relaying in Nakagami-fading environments," IEEE Trans. Wireless Commun., vol. 6, pp. 4256-4262, Dec. 2007.

[18] M. K. Simon and M.-S. Alouini, "A unified approach to the performance analysis of digital communications over generalized fading channels," Proc. IEEE, vol. 86, pp. 1860-1877, Sep. 1998

[19] I. S. Gradshteyn and I. M. Ryzhik, Table of Integrals, Series and Products, 6th ed. New York, NY, USA: Academic Press, 2000 